\title{
Extracting Hidden Hierarchies in 3D Distribution Networks
}

\author{
Carl D. Modes, ${ }^{1, *}$ Marcelo O. Magnasco, ${ }^{1}$ and Eleni Katifori ${ }^{2,3}$ \\ ${ }^{1}$ The Rockefeller University, New York, New York 10065, USA \\ ${ }^{2}$ Department of Physics and Astronomy, University of Pennsylvania, \\ Philadelphia, Pennsylvania 19104, USA \\ ${ }^{3}$ Max Planck Institute for Dynamics and Self-Organization (MPIDS), 37077 Goettingen, Germany \\ (Received 21 October 2014; revised manuscript received 8 April 2015; published 20 July 2016)
}

\begin{abstract}
Natural and man-made transport webs are frequently dominated by dense sets of nested cycles. The architecture of these networks, as defined by the topology and edge weights, determines how efficiently the networks perform their function. Yet, the set of tools that can characterize such a weighted cycle-rich architecture in a physically relevant, mathematically compact way is sparse. In order to fill this void, we have developed a new algorithm that rests on an abstraction of the physical "tiling" in the case of a twodimensional network to an effective tiling of an abstract surface in 3-space that the network may be thought to sit in. Generically, these abstract surfaces are richer than the flat plane because there are now two families of fundamental units that may aggregate upon cutting weakest links - the plaquettes of the tiling and the longer "topological" cycles associated with the abstract surface itself. Upon sequential removal of the weakest links, as determined by a physically relevant edge weight, such as flow volume or capacity, neighboring plaquettes merge and a new tree graph characterizing this merging process results. The properties of this characteristic tree can provide the physical and topological data required to describe the architecture of the network and to build physical models. The new algorithm can be used for automated phenotypic characterization of any weighted network whose structure is dominated by cycles, such as mammalian vasculature in the organs or the force networks in jammed granular matter.
\end{abstract}

DOI: 10.1103/PhysRevX.6.031009

\section{INTRODUCTION}

Complex networks are a pervasive presence in both modern technology and the natural world, with examples as widely varied as transportation networks, the internet, mammalian vasculature, the neuronal connections in the brain, load-bearing architecture, river deltas, venation in plant leaves, and even slime molds. Driven largely by the explosion in importance of the world wide web, academic interest in the associated study of complex networks has risen in prominence as well in recent years, with the introduction of important new classifying ideas such as "small-world" [1] and "rich-get-richer" [2] network topologies. Inspired by the anything-goes hyperlinked structure of the internet itself, many of these studies concentrate only on the topological and graph-theoretic properties of general classes of complex networks with unrestricted node degree.

On the opposite end of the spectrum from these hyperlinked networks are those systems which naturally live in two dimensions, such as river deltas or plant-leaf venation.

*cmodes@rockefeller.edu

Published by the American Physical Society under the terms of the Creative Commons Attribution 3.0 License. Further distribution of this work must maintain attribution to the author(s) and the published article's title, journal citation, and DOI.
Subject Areas: Biological Physics, Complex Systems, Computational Physics

By virtue of their embedding in low-dimensional space, these networks necessarily feature restricted degree distributions where each node connects to a specified number of neighbors, in contrast to more general, abstract networks. Much recent work has also served to elucidate matters here as well, from a comprehensive sedimentary modeling of the river delta structure and evolution $[3,4]$ to the construction of a hierarchy-sensitive and geometry-independent topological characterization of the plant leaves [5-7]. Other methods that rely on local geometric cues to characterize leaves, road networks, and crack patterns [8], or topological characterization of networks of epithelial contacts [9], have also been explored.

However, there is an important class of complex networks that lie somewhere in between these two extremes, sensitive to geometric embedding in space and equipped with restricted degree distributions, yet too complex in structure to live entirely in the plane. This intermediate class has seen relatively fewer recent advancements, with most activity centered around mapping and descriptive efforts of certain functionally relevant network phenotypes $[10,11]$, though even simple vascular cartography is fraught with difficulties at the mesoscale. Several members of this intermediate class are networks where a deeper understanding would have far-reaching biomedical implications, such as vascular or neural nets, so the impact of a proper 
tool to characterize them would be immense, opening the door to predictive modeling of function and diseaseprocess-driven malfunction [12]. Furthermore, powerful and effective quantitative descriptors are a necessary first step to both disentangling the network architecture's role during development-of itself and the surrounding tissue - and to advanced, three-dimensional artificial organ synthesis.

The idea behind our cycle-hierarchy sensitive characterization is based on one of the recent methods developed to describe distribution networks in two dimensions [5,7]where adjacent cycles in the network are allowed to sequentially merge into larger and larger cycles. The order of merger and relative location of these cycles can be mapped onto a bifurcating tree graph, and a number of simple statistical measures of this tree are then easily accessible and contain information about the original network. For a distribution network-or, more generally, any weighted network - that lives in three dimensions, however, we are presented with an imposing hurdle at the very beginning of the process: What does it mean for two cycles to be adjacent? Our method solves this question by imagining that the network lives on a topologically nontrivial surface in space and represents an effective tiling of that surface, from which cycle adjacency and the rest of the prescription from the two-dimensional case naturally follows.

We begin in Sec. II with a quick primer and refresher on some classical results of graph theory necessary to understand the core of our algorithm. We then lay out the machinery of our characterization and quantification algorithm in Sec. III, and we use it on several examples of abstractly interesting or physically relevant surrogate networks in Sec. IV. We discuss implications and future directions in the concluding Sec. V.

\section{BRIEF GRAPH THEORY REFRESHER}

In the interest of providing a quick reference and explanation of some of the classical graph theoretic concepts integral to this work, this section covers the concepts of a graph's cycle space and graph embeddings and genus. This section is intended as a simple review; a reader comfortable with these topics should feel free to skip ahead. Our new cycle-coalescence algorithm is presented in Sec. III.

\section{A. Cycle space}

Since our ultimate target is to construct a characterization of cyclic paths in distribution networks, it will be helpful to make contact with the mathematical structures that cycles in a graph may be endowed with. For the remainder of this work, we assume that all graphs are simple graphs; i.e., no pair of vertices may be directly connected by more than one edge. Given such an arbitrary graph, it turns out that the superset of all cyclic paths without repeated traversal of the same edge can be thought of as a vector space, known as the cycle space [13]. In this formalism, the individual elements (i.e., the "vectors") are sets of cyclic paths represented as the entire graph, with each edge assigned a 0 if it is not traversed in a cycle and a 1 if it is. The dimensionality of this vector space is accordingly less than the number of edges in the graph. The vector addition is defined as a simple edgewise addition of the graph representatives for each vector being added. Furthermore, the cycle space in its simplest form is defined over $\mathbf{Z}_{2}$, so if an edge is used in each member of the binary summand it is not used in the sum. Thus, for example, if one were to add two adjacent cycles in a graph, the result would be the larger, boundary cycle.

Why do elements defined on a graph in this way, equipped with this addition rule, constitute a vector space? Much of this is down to the simplicity of working over $\mathbf{Z}_{2}$-scalar identity, compatibility between the multiplication and addition in $\mathbf{Z}_{2}$, and distributivity of the scalar multiplication with respect to both the vector addition and the field addition all follow trivially from $\mathbf{Z}_{2}$ 's simple twoelement structure. Meanwhile, the associativity and commutativity of vector addition are satisfied by construction, leaving only the specification of an identity element and inverse elements under the vector addition for any member of the vector space. Clearly, a graph adorned with zeroes on every edge, a null cycle, is the appropriate identity vector. Again appealing to the simplicity of $\mathbf{Z}_{2}$, it is clear that every vector in the cycle space is its own inverse since each edge with a zero will remain zero and each edge with a 1 will be set to zero upon the addition of a vector to itself. Note that, further owing to that same simplicity of working over $\mathbf{Z}_{2}$, the cycle space is not equipped with an inner product.

Having established that the cycle space is truly a vector space, it is a natural next step to discuss some of its relevant properties. As all vector spaces must be, the cycle space is equipped with a basis, and as it happens, there also exists a convenient way to generate such a basis. By choosing any spanning tree-a subgraph that contains every vertex but no cycles [14] —on the graph and adding a single further edge to the tree, one sees that precisely one cycle is created by the union of the spanning tree with the chosen extra edge after pruning all extraneous tips (or "leaves") left over from the spanning tree. Furthermore, choosing a different extra edge produces a different cycle. The set of all cycles created in this way constitutes a basis for the space [15]. Clearly, if one had chosen a different spanning tree to begin this process, then a different basis would result, but despite the large number of spanning trees available for an arbitrary graph-typically exponential in the number of vertices and possibly worse [15] — bases generated in this way are referred to as fundamental cycle bases [13] and are actually quite special and in some cases comprise only a small portion of the total number of possible bases. Note that this 
procedure provides a direct way to compute the dimension of the cycle space: For a graph with $v$ vertices and $e$ edges, a spanning tree must use precisely $v-1$ edges, leaving $e-v+1$ edges to create the fundamental cycles. Hence, the dimension of the cycle space for an arbitrary graph is $e-v+1$.

Furthermore, among this massive collection of possible bases for the space, one may look for special bases that optimize certain simple properties. For example, there exists a minimum weight basis that contains the collection of basis vectors whose combined sums over the edge weights is as small as possible. In the event that every edge is simply assigned a weight of 1 , then the minimum weight basis corresponds to the basis that collectively uses as few edges as possible. This particular minimum weight basis suggests a connection to tilings - if a graph is a simple tiling of the plane, such as a checkerboard, then it is likely that not only is the set of individual tiles a basis for this graph's cycle space but that the basis is minimum weight in the way described above. There is one subtlety here that must be pointed out, however-if the boundary cycle (i.e., the cycle that remains upon summing all the tile basis vectors) has fewer edges than one of the actual tiles, then it is included in the minimum weight basis in the place of the largest tile. There is a well-defined sense in which it is actually more appropriate to think of these plane tilings as tilings of the sphere instead, wherein the boundary cycle is just another tile. In this setting, the minimum weight basis is exactly the set of all the tiles but the largest. To push this analogy further, though, we must first confront the separate subject of graph embeddings.

\section{B. Graph embeddings}

As outlined above, certain special graphs may be thought of as tilings of the plane. This occurs when a graph may be represented in the plane without any edges crossing one another; graphs that have this property are known as planar graphs. All representations of a planar graph do not necessarily sit in the plane without edge crossings-just one such representation will suffice. As an example, the complete graph on four vertices, $K_{4}$, is not embedded in the plane in its most traditional representation, but it is planar nonetheless [see Fig. 1(a)]. A randomly chosen graph with many vertices, however, is vanishingly unlikely to be planar [16] — indeed, one only needs five vertices for nonplanar graphs to begin to appear as $K_{5}$ is one such, and they rapidly dominate as more vertices are added.

On the other hand, all graphs may embed into space with no edge crossings. The simplest way to see that this is true is through a process known as the book embedding [17]: Imagine placing all vertices on a line in space, to be thought of as the spine of an open book. Then every edge may be placed by connecting its two end-point vertices without the possibility of intersecting any other edge if each edge has an (infinitely thin) page of the book to itself. Since such an (a)
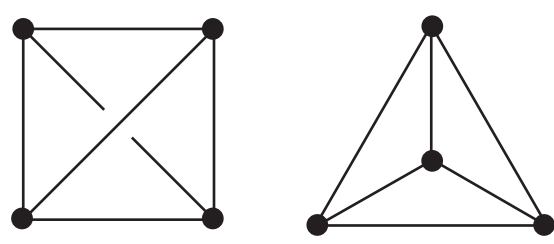

(b)
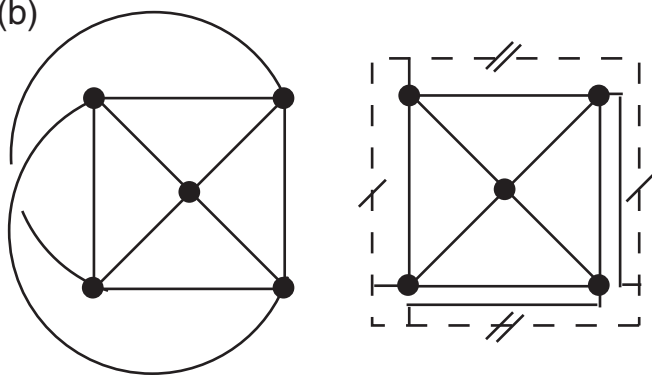

FIG. 1. Some simple examples of graph embeddability, both planar and otherwise. (a) The complete graph on four vertices, $K_{4}$, is not embedded in the plane in its most traditional representation (left) but is planar nonetheless (right). (b) The complete graph on five vertices, $K_{5}$, is not planar (left). However, this graph can be represented without edge crossings on a torus (right).

embedding is always possible, it follows that embedding a graph without edge crossings on a complicated surface is also always possible-simply construct a surface with characteristic distances smaller than the vertex separations and follow the edges of the book embedding. This surface is, of course, extremely complicated, with genus, $g$ - the number of "holes" or "handles" in the surface — of order the number of edges in the graph. However, since a graph may always sit in a surface in space, it is well defined to ask what the simplest (i.e., lowest genus) surface is that can accommodate an embedding of a particular graph. As an example, consider again the simplest graph that is not planar, $K_{5}$-as shown in Fig. 1(b), this graph can be represented without edge crossings on a torus. Unfortunately, in general, the problem of determining the graph genus for an arbitrary graph is NP hard [18]. However, the existence of a simplest topological surface on which a graph may be thought to tile is all that is needed to proceed.

Since planar tilings represented a simple example in the previous discussion of the cycle space, it is worth pointing out what happens in the more general, nonplanar case. As just discussed, every graph may be represented as a tiling on a surface of some genus, and clearly, a minimum weight basis on the edge count must at least include all the tiles (except the largest one, for the same reasons as above). But is there anything new? We know that the number of elements of the basis must be $e-v+1$, and we also know that the number of elements that came from tiles is 


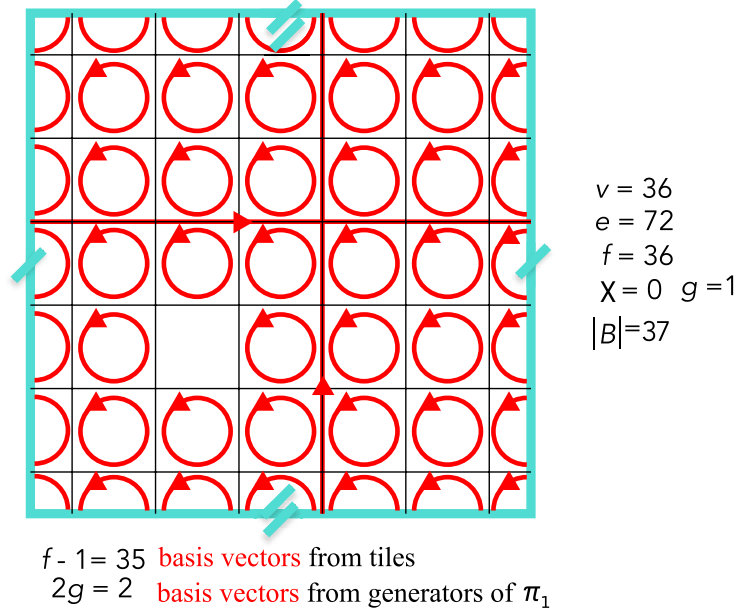

FIG. 2. Tiles and topological cycles of a square lattice on a toroidal topology $(g=1)$. Opposite sides of the lattice are identified as shown.

$f-1$ for a tiling with $f$ faces or tiles. Putting these two facts together with the formula for the Euler character in terms of the genus, $v-e+f=2-2 g$, yields a clear overage due to the topology:

$$
e-v+1=(2 g)+(f-1) \text {. }
$$

Indeed, the promotion of the embedding surface to one with genus necessitates some new basis elements: the generators of the fundamental group of the surface $[19,20]$, which encodes the contractibility of families of paths on the surface. There are precisely $2 g$ such generators (see Fig. 2). As can be seen by inspection of Fig. 1(b) or 2, no sum of the simple tiles can give a (single) cycle that winds across one of the two periodic boundaries, and yet these cycles clearly exist in the graph, so the cycle basis must generate them somehow. The inclusion of these new elements resolves this issue.

We therefore expect our cycle basis to consist of $f-1$ tiles and $2 g$ topology generators. For graphs that can be embedded on toroidal surfaces with "fat" handles, the sum of edges of the $2 g$ topology generators should all be greater than the sum of edges of the tiles, thus allowing us to easily identify the tiles from the generators. However, such a clear size separation of the tiles and generators is not always guaranteed: Otherwise, direct reconstruction of the graph embedding would be possible in polynomial time. We construct our algorithm and discuss some of the expectations and statistics related to this nonguarantee and the corresponding ability to extract information without explicitly constructing the embedding in the next section.

\section{CYCLE-COALESCENCE ALGORITHM}

We now describe how the vectors of the minimal basis are hierarchically added by the cycle coalescence algorithm. The algorithm consists of three separate parts and eventually generates a characteristic, linkage tree. We assume that the networks that are being analyzed have no loops (edges that terminate at the same node), double edges or bridges, i.e., that they constitute simple graphs that are at least 2-connected.

First, we describe how to obtain the minimum weight basis over the edge count of the unweighted graph, hereafter termed "minimal basis" to avoid confusion. Second, we show how to sequentially merge the identified tiles and construct the characteristic tree. Last, we discuss various metrics one can use to describe the structure of the characteristic tree, and what these metrics mean for the architecture of the original graph.

\section{A. Finding the minimum weight cycle basis}

There are a number of polynomial time algorithms that can be used to construct the minimum weight basis [21]. Starting from an arbitrarily chosen node in the unweighted network, we find the minimal spanning tree-equivalent to executing a breadth-first search over the graph-and determine the fundamental cycle basis associated with that node. We repeat for every node of the network, in this way generating a set $S$ containing all the unique vectors of the fundamental cycle bases associated with each node. We sort the set $S$ based on vector size in an ascending order and start building a minimal basis $B$ bottom up, by sequentially adding vectors from smallest to larger. When two vectors in $S$ are degenerate, their respective order in the sorted set is determined at random. Before any vector $b_{i}$ is added to $B$, we check if that vector is linearly independent from the vectors already added to the set. If the vector depends linearly on the vectors already added to the set, that vector is discarded and the next vector $b_{i+1}$ is checked for linear independence. The set $B$ closes and becomes a real basis when no new vector can be added. However, we do not need to check every vector in $S$-we know that the number of elements in the basis is $e-v+1$, so the search is terminated when the cardinality of $B$ becomes $e-v+1$. Examples of fundamental basis vectors in $S$ and minimal basis vectors in $B$ can be seen in Figs. 3 and 4(a). In the case of degeneracies, i.e., the existence of more than one minimum basis, the output minimum basis might be dependent on the chosen algorithm. However, the statistical properties of the final characteristic tree are generally robust and, with the exception of some singular cases, do not depend sensitively on the exact basis. Furthermore, we expect that most of the degeneracy will be confined to cycles representing topology generators not tiles, further minimizing their impact on the final output.

\section{B. Constructing the characteristic tree}

The minimum weight basis $B$ contains only topological information of the original graph, as in constructing $B$ we ignored the weights of the individual edges. The cycle 
(a)

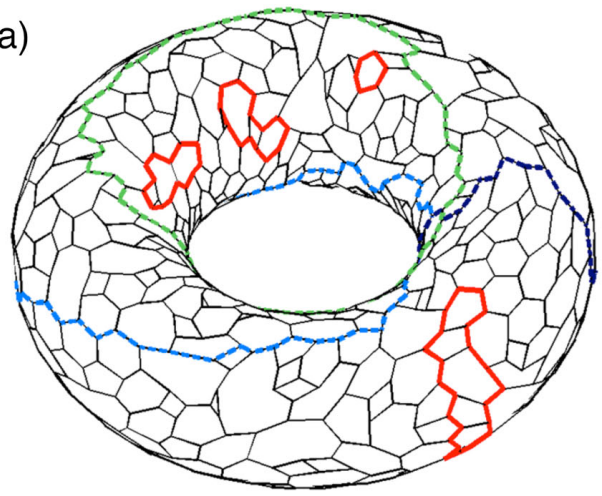

(b)

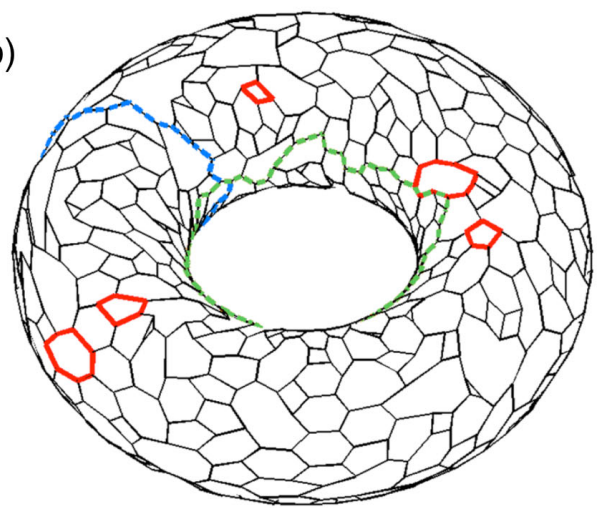

FIG. 3. A graph embedded on a toroidal surface. Highlighted are example basis vectors from (a) a fundamental cycle basis and (b) a minimum weight basis. Red: Some representative tiling basis vectors. Blue and green: Basis vectors that correspond to the $2 g=2$ generators of the fundamental group of the torus.

coalescence algorithm integrates the structural information carried by the edge weights in the construction of the characteristic tree. The algorithm begins with a set of vectors $B_{\text {iter }}$ identical to the basis $B$. The set $B_{\text {iter }}$ will be updated (vectors added and removed) throughout the algorithm.

To begin, we identify the edge $e_{i}$ of the graph with the smallest weight. We then locate the basis vectors $\left\{b_{k}\right\}$ in $B_{\text {iter }}$ that contain that edge. An example is shown in Fig. 5(a), where basis vectors $B E F C, B E H A$, and $B E H G$ all pass through edge $B E$. The two shortest vectors that contain that edge are added as described in Sec. II, creating a new cycle. As described below, this choice will statistically preferentially merge tile cycles over generator cycles, creating a steadily ever-more coarse-grained tiling of the abstract surface. This new cycle is added to $B_{\text {iter }}$, and the two original vectors are removed. In our implementation, degeneracies are again resolved here by random choice within the degenerate vectors. In Fig. 5(a), the addition of $B E F C$ and $B E H A$ results in the cycle $F C B A H E F$, color-coded in green. The algorithm proceeds by identifying the next smallest edge that is utilized by at least two vectors in $B_{\text {iter }}$ and iteratively repeating the process until there is only one cycle left, as in the bottom of Fig. 5(a). (a)

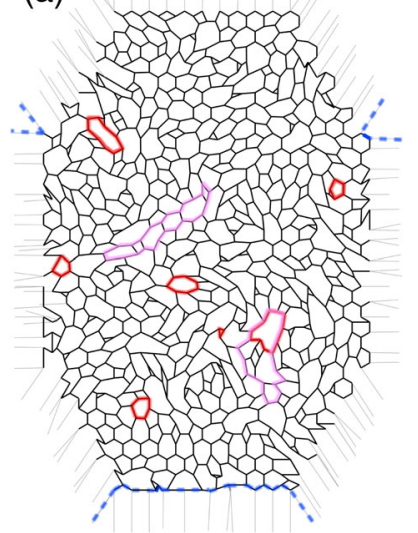

(b)

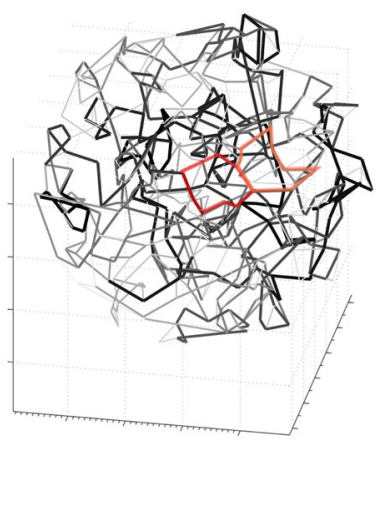

FIG. 4. (a) Graph embedded on a two-holed torus, schematic. Periodic boundary conditions connect opposite sides of the octagon. Highlighted are some example tiling basis vectors from a fundamental cycle basis (magenta) and from the minimum weight basis (red). The blue curve highlights one of the $2 g=4$ vectors in the minimal basis corresponding to the generators of the fundamental group of the two-holed torus. (b) A 3D graph with an unknown embedding. Identification of tiling versus generator basis vectors is not straightforward anymore. Highlighted with red and orange are two vectors in the minimum weight basis that share an edge.

This cycle-coalescence algorithm can be represented with an unweighted, bifurcating tree whose nodes represent cycles of the original graph and the links connect cycles that are connected via cycle addition operations. In particular, the basis vectors in the initial set $B$ are the leaf (terminal) nodes of the tree, represented by the six nodes on the top-right panel of Fig. 5(a). When two cycles are added, they result in a new cycle, represented by their parent node in the bifurcating tree. Hierarchically joining the cycles based on the sort order of the edges thus results in a tree whose bifurcation statistics capture the structure of the nested cycles of the network. An architecture dominated by highly nested cycles will produce a close-to-perfect binary tree, whereas a more disordered architecture will produce an asymmetrical tree [22].

The information encoded in this characteristic tree does not depend on the geometric location of the nodes nor the exact value of the edge weights; it only depends on the network connectivity and sort order of the edge weights. In fact, as shown in the example of Fig. 5(b), the characteristic tree is invariant under any node movement or edge-weight change (provided that the sort order is maintained). The characteristic tree is thus an ideal tool to describe structural information about the network not captured by widely used metrics such as edge-weight distributions or weighted or unweighted degree distributions [23,24].

The computational complexity of the algorithm is dominated by finding the minimum weight basis. An optimized implementation is capable of running in polynomial time $O\left(e^{2} v / \log v\right)$. 
(a)
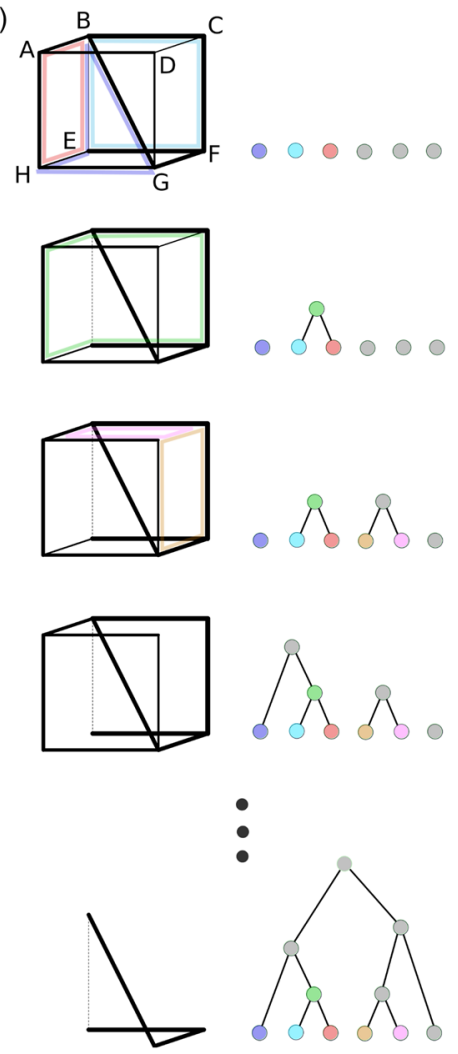

FIG. 5. (a) Schematic of the cycle-coalescence algorithm applied to a simple 3D graph. The cycle coalescence proceeds from top to bottom. At each step, the next weakest link is cut, and the two cycles that bordered that link coalesce into one. On the right, we show the characteristic tree being progressively built, with each node representing a cycle in the network. To aid the eye, some cycles that participate at that stage of the algorithm are highlighted in color, and the corresponding nodes of the characteristic tree are correspondingly color-coded. (b) Schematic of degenerate graphs that produce the same characteristic tree. The cycle coalescence is blind to geometry and exact weights of links.

\section{Statistical arguments for the cycle-merger choice}

In the above-described cycle-coalescence algorithm, we have assumed that the appropriate choice of cycle space vectors to merge upon the cutting of an edge in the graph is simply the two shortest cycles by number of edges traversed. The rationale for making this choice is that in the abstract representation of the graph as a tiling of a potentially complex surface living in three dimensions, we desire to merge adjacent tiles rather than cycles that have arisen because of the nontrivial topology of the surface. The intuition is that, generically speaking, "real" tiles should be shorter than cycles that traverse entire "handles" of the surface, especially as the number of vertices in the graph tends to infinity for controlled degree distributions. This intuition can be made concrete in a statistical sense, but to do so, we must consider how a basis vector of the cycle space that arises from topology and not from tiling might be shorter than one that represents a tile and how likely it is that this occurs. In order to ensure that the graphs we consider comply with the need for a controlled degree distribution, we restrict ourselves in what follows to the simplest such family: 3-regular graphs, where each vertex joins three edges. Despite this choice, we do not lose much for relevance, as nearly all biological and most physical distribution networks are of this type or close to it with scarce trifurcations or higher-order branchings, having developed primarily through multiple stages of binary branching and/or tip-to-channel reconnections [25]. Additionally, the form if not the specific detail of the following arguments will apply to other families of graphs with different, controlled degree distributions.

Before proceeding, it is useful to establish a "basal" 3-regular tiling from which we can explore more complicated variants. We leave the genus of the surface, $g$, as a free parameter. A tiling of such a surface satisfies the familiar formula for the Euler character in terms of the genus invoked earlier: $2-2 g=v-e+f$. Furthermore, for a 3 -regular graph, $v$ and $e$ are related by $e=3 v / 2$. How does the genus affect the average number of edges per tile, $|p|$ ? Since every face shares each of its edges with another face, we must have $|p| f=2 e$, and hence,

$$
|p|=\frac{6 v}{4+v-4 g} .
$$

It is therefore clear that so long as the number of vertices in the graph is much larger than the genus of the tiled surface, we may imagine our basal tiling as a simple hexagonal net with a handful of isolated heptagonal [necessary by Eq. (2) when $g>1$ ] or pentagonal tiles (when $g=0$ ) due to the topology of the surface. As an aside, in the case of a spherical topology, this is the source of the icosahedrally symmetric pentagonal defects one encounters in, for example, viral capsids, fullerene, geodesic domes, and soccer balls [26]. Note that for a simple toroidal topology with $g=1$, the basal tiling is a perfect hexagonal crystal [Fig. 6(a)].

Having established our set of basal tilings, we must consider the fundamental unit of perturbation away from these tilings that still respects the topological relationship discussed above. In particular, we may not freely insert tiles with more or fewer sides than the average given the genus and number of vertices - we must at least replace a pair of hexagons from the basal tiling with a pair of polygons whose total number of sides sums to 12 to ensure that we satisfy the necessary $|p|$. A positive departure from 6-sidedness in this context is known as a negative disclination defect and a negative departure as a positive disclination defect. In this language, the necessary condition of a perturbation is that it has no net disclination charge. This condition is not, however, sufficient: A disclination dipole produces a dislocation defect on this 
(a)
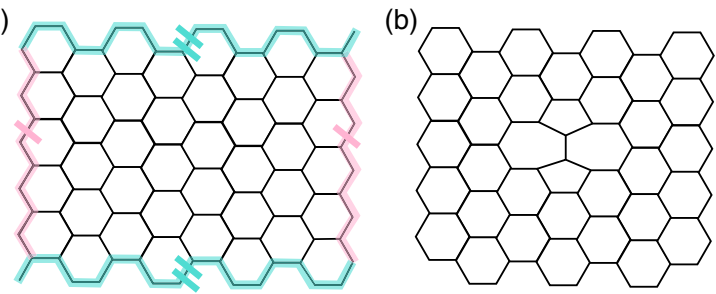

(c)

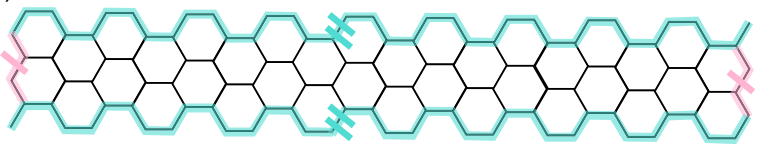

(d)

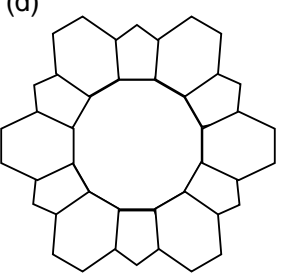

(e)

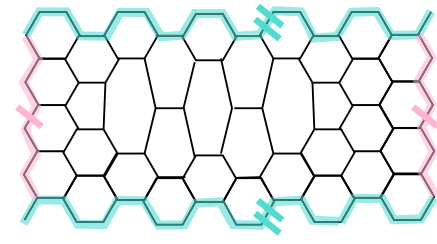

FIG. 6. (a) Honeycomb lattice on a toroidal topology. Opposite sides are identified as shown. (b) T1 topological rearrangement on a honeycomb lattice. (c) One-holed torus with a thin handle. (d) A tile with a large number of sides as a result of condensation of disclination charges. (e) Varicosity as a result of a condensation of like-charged dislocation defects. Opposite sides are identified as shown, resulting in the topology of a one-handle torus.

crystalline background, and since our tiling exists on a compact, closed surface without boundary, it cannot escape to infinity nor can it simply end on a boundary - it must be terminated by a second disclination dipole. These conditions together, that there is neither disclination nor dislocation charge, are necessary and sufficient for the production of a self-consistent perturbation. These perturbations take the form of a disclination quadrupole, also known as a T1 topological rearrangement [27] [Fig. 6(b)].

With our basal tilings and working perturbative units in hand, we may now tackle the question before us: How can the assumption that tiles are "shorter" than topological cycles fail? One possibility is that one of the directions around a handle is very short [Fig. 6(c)]. For fixed $v$, a surface may be represented as a $4 g$-gon with appropriate sides identified [28]. In the case in which all handles are of the same thickness and length, this $4 g$-gon becomes regular, with side lengths that scale as $v^{1 / 2} / g$. Unless $g$ is very large relative to and scales linearly with $v$, the number of cases where a shrinking handle is still "thicker" than the circumference of a tile is of order $v^{1 / 2}$, and hence a problem of this type is encountered with likelihood that scales as $1 / v^{1 / 2}$. Note that an extrinsically growing genus does represent a real issue here, and we conclude that our algorithm is less likely to provide meaningful insight for

ordered structures with small, simple unit cells since the genus in these cases is large relative to $v$ and grows linearly with the addition of further unit cells to the crystal.

Turning to our quadrupolar perturbations, we see that another potential problem arises not when a handle traversal becomes short but when a tile becomes large: Condensation of one of the disclination charges upon repeated perturbations can lead to a tile with a large number of sides [Fig. 6(d)]. With $f=2 e /|p|$ faces in a 3-regular graph, let us further assume that the genus is low enough relative to $v$ that $|p|$ is near 6 , in other words, that the basal tiling is still a hexagonal net with sporadic heptagons. Since we have already discounted very high genus scenarios because of the above reasoning for handle thinness cases, little more is lost here. With $|p| \approx 6$, we have $f \approx v / 2$ and $e=3 v / 2$. There are precisely as many $\mathrm{T} 1$ topological rearrangements available as there are edges in the graph, and those that increase the number of sides of a given tile occur by selecting an edge that shares a vertex with the tile but is not an edge for it. Since the graph is 3-regular, there is only one such edge per vertex. Therefore, the number of perturbations that increase the number of sides for a given tile with $p$ sides is simply $p$. Note that the same number of perturbations exist that decrease the number of sides for that tile. The likelihood of significant condensation is clearly very low, occurring at a rate

$$
P(p=N>7) \sim \frac{2(N-1) !}{6 !\left(\frac{3 v}{2}\right)^{N-7}}
$$

for magnitude $N-6$ perturbations, and it may be safely ignored.

Finally, there is the possibility that a handle experiences varicosity. Unlike in the first case considered where the handle thickness is simply too small across the entire handle, when a handle experiences varicosity, the handle thickness varies as a result of a condensation of likecharged dislocation defects and becomes locally too thin as a result [Fig. 6(e)]. $L$ consecutive T1 events must occur in exactly the right position-overlap of neighboring 5-7 pairs - simply to open up a single dislocation "scar" of length $L$, with likelihood scaling as $1 / v^{L}$. This must occur several more times, and the opened scars must align, and their projection onto the direction normal to the handle traversal must result in concurrent overlap for there to be any chance of generating a cycle shorter than the hexagonal and heptagonal tiles that are the primary constituents of the graph. This eventuality may be safely ignored, too.

Since, as we discuss in the following subsection, the characteristic tree will ultimately be subject to its own round of statistical analysis, there is even more built-in statistical robustness than even the above arguments indicate. Even if the characteristic tree fails to accurately recapitulate the "true" tile coalescence pattern, this failure 
will be isolated to a handful of specific nodes and will have a minimal effect on statistical measures of the tree.

\section{Quantifying the characteristic tree}

The characteristic tree provides a convenient way to analyze the architecture of a complex weighted network that is composed of cycles. The topology of the characteristic tree reflects the hierarchy of cycle nesting in the original weighted graph. A detailed discussion on how different tree bifurcation statistics map to various graph architectures and how one can use the characteristic tree to quantify the degree of nestedness of planar graphs and to analyze their weighted topology can be found in Ref. [5]. In summary, the more balanced (low height) the tree is, the more highly nested the original graph. High weight cycles are subdivided iteratively by smaller weight edge, creating a cascade of hierarchically nested cycles. On the other hand, high-height, unbalanced trees typically represent graphs where smaller weight cycles are added sequentially on the backbone of bigger cycles, creating an architecture that is less hierarchically organized. Examples of graphs with high hierarchical organization are shown on the left columns of Fig. 7(a) (in 2D) and Fig. 7(a) (in 3D). For comparison, on the right columns, we show graphs whose cycles are not hierarchically nested.

To analyze the characteristic tree, we need to assign a number to each tree architecture. There are several schemes that have proven quite useful in quantifying several aspects of the tree: Horton and Strahler numbers, the tree height, etc. [29-31]. Each scheme has its advantages and disadvantages, and discriminatory power that focuses on different aspects of the architecture. In this paper, we use an adapted version of the partition asymmetry, as introduced in Ref. [31]. The partition asymmetry is a metric that characterizes the overall topological structure of a binary tree and quantifies the difference in size (number of leaf nodes) between the two subtrees that stem from a tree vertex. We define the partition asymmetry $a(j)$ of a bifurcation vertex $j$ as

$$
a(j)=\frac{s_{j}-r_{j}}{s_{j}+r_{j}-1},
$$

with $s_{j}>r_{j}$ and $s_{j}+r_{j} \geq 2$. The parameters $r_{j}$ and $s_{j}$ are the degrees of the two subtrees at partition $j$. The degree of a (sub)tree is defined here as the total number of the leaf nodes (terminal segments) of that (sub)tree.

The partition asymmetry $a(j)$ provides a number for each vertex of the characteristic tree that quantifies the degree of hierarchical organization in the loop nestedness of the original graph. A tree that is balanced and corresponds to a hierarchically nested graph will have many low-asymmetry vertices. Conversely, a graph that is not hierarchically nested will produce a characteristic tree with many vertices that have high asymmetry. Thus, comparing
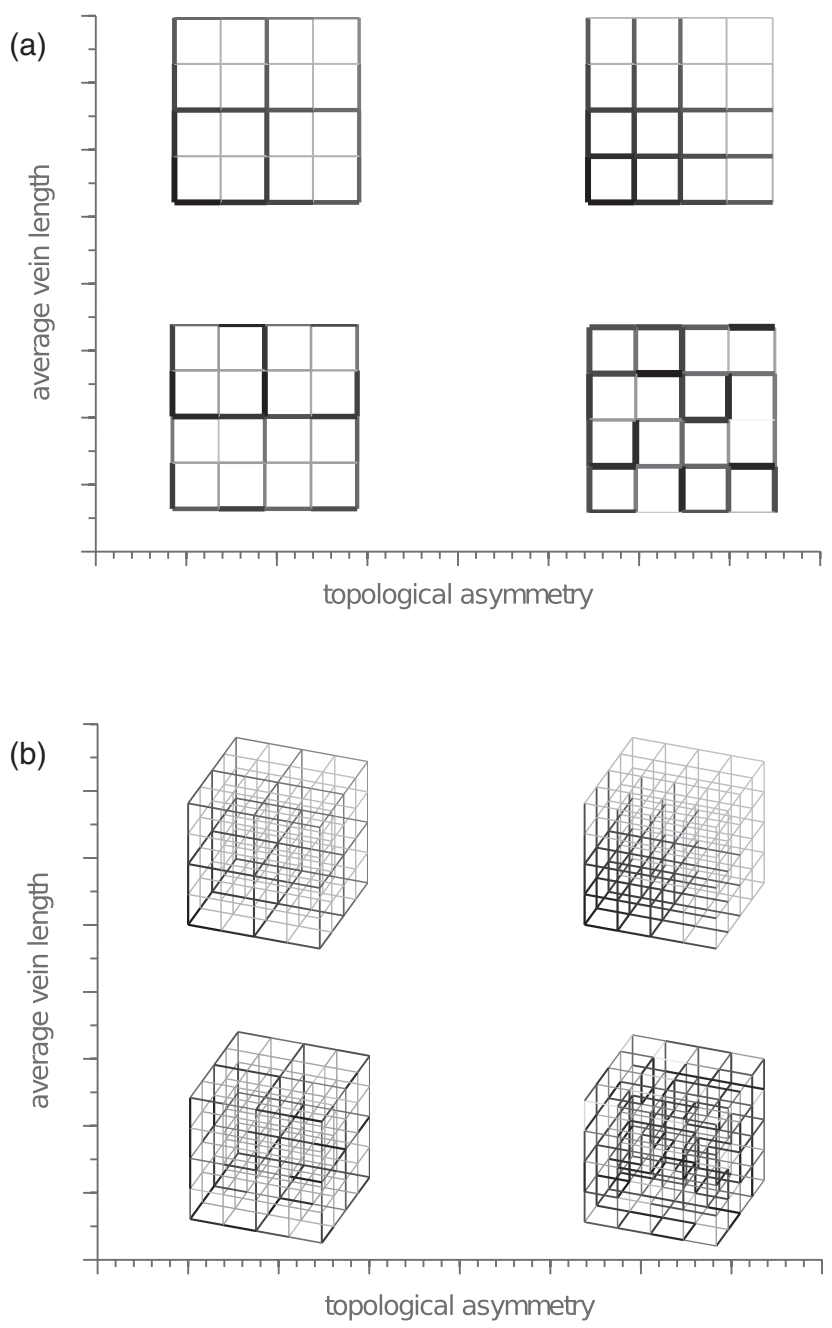

FIG. 7. Average vein length versus topological asymmetry for toy graphs in (a) 2D and (b) 3D.

the distribution of partition asymmetries of two graphs can be a metric on how statistically similar the architecture of those two graphs is.

In order to characterize the architecture of the graph, instead of pairwise comparison of asymmetry distributions, we define the topological asymmetry $A$ of a graph, by measuring the percentage of vertices in the tree that have asymmetry higher than 0.95 ,

$$
A=p[j \mid a(j)>0.95] .
$$

The higher the topological asymmetry of the graph, the less hierarchically nested the graph is.

Each node in the characteristic tree represents a cycle in the original graph, and the subtree that is rooted at that node encompasses information about the architecture of part of the graph "contained" in the cycle. The higher the degree of the node in the characteristic tree, the bigger the part of the graph represented in the subtree. Thus, as the tree contains many more nodes far away from the root than close to it, the 
distribution of the partition asymmetry is dominated by the architecture at the small length scales. There are many more nodes close to the leaves of the characteristic tree, meaning that any partition asymmetry average, unless weighted in favor of high-degree nodes, will be dominated by the architecture at the short length scale. If the architecture of two graphs is statistically similar at small length scales, the topological asymmetry $A$ will not have enough discriminatory power to distinguish them. We need a second quantity that characterizes some of this missing information.

The average vein length $L$ is a topological length that quantifies the average length of the "veins" in the weighted graph (not the characteristic tree itself). It is calculated by constructing trails where the edge weight declines monotonically. Starting from an initial link $e_{1} \equiv\left\langle i_{1} j_{1}\right\rangle$ between nodes $i_{1}$ and $j_{1}$ [first link of the trail $t=\left(e_{1}\right)$ ], we identify all the links $\{e\}$ that are adjacent to it (share the node $i_{1}$ or $j_{1}$ ) and have weight smaller than or equal to the weight $w\left(e_{1}\right)$ of edge $e_{1}$. We choose the link with the maximum weight from the set $\{e\}$, which we will call $e_{2}$, and add it to the trail, which now becomes $t=\left(e_{1}, e_{2}\right)$. We now repeat the process for $e_{2}$ [identify all links that are adjacent to $e_{2}$ with weight smaller than $w\left(e_{2}\right)$ and choose the maximum] and iterate. The algorithm is stopped when the set of links that have weight smaller than the weight $w\left(e_{k}\right)$ of the last link $e_{k}$ in the trail is empty. The length of the trail $t=\left(e_{1}, e_{2}, \ldots, e_{k}\right)$ associated with edge $e_{1}$ is $l\left(e_{1}\right)=k$. We repeat the process starting from every link of the graph, in this way associating a trail length $l(e)$ with every link $e$. The average vein length is defined as

$$
L=\frac{1}{|e|} \sum_{e} l(e) .
$$

Note that it is generally not the case that $L$ and $A$ may be varied independently by picking edge weights "by hand," especially if the embedding structure is unknown.

To visualize the interpretation of the average vein length $L$ and topological asymmetry $A$, in Fig. 7 we present a series of toy graphs occupying the corners of an $L$ versus $A$ plot. Figure 7(a) presents the easier-to-visualize 2D planar graphs, whereas Fig. 7(b) showcases some equivalent examples of 3D graphs. Moving from left to right on the $x$ (asymmetry) axis, the nestedness of the cycles becomes less pronounced. Moving from bottom to top on the $y$ (vein length) axis, long "highways" become more pronounced. The top left graph is a highly nested and high-vein-length graph, whereas the bottom right is a graph where the weights have been distributed at random. We provide the algorithm to extract the topological asymmetry and average vein lengths for planar graphs in Ref. [32].

Here, we need to stress that the topological length $L$ and the topological asymmetry $A$ are just two of the many ways one can measure the architecture using purely topological information. Equivalent choices could be weighted asymmetries with weights that favor nodes close to the root of the characteristic tree, for example- as pointed out above, the statistical tools available for analysis of a tree graph are manifold. The best choice can and should depend on the nature of the data being analyzed.

\section{EXAMPLES AND SURROGATE DATA}

To test our new characterization tool, we computer generated a series of weighted networks produced by a number of distinct generation protocols. We chose these generation protocols so that the produced networks would be statistically indistinguishable under many widely used network metrics, to demonstrate the power of the cycle-coalescence algorithm. We also chose generating functions with some biological relevance to vascular networks. The degree of all the networks was strictly equal to 3 , as would typically be the case in a natural transport network — as pointed out above—-such as plant or animal vasculature [25].

\section{A. Example networks}

For our example networks, we considered two types of underlying topologies. In the first, the graph, by construction, could naturally be embedded on a two-holed torus. The original graph was produced by generating a regular hexagonal grid on a plane, applying the appropriate periodic boundary conditions to create a two-holed torus and finally applying a random series of $\mathrm{T} 1$ transformations to introduce lattice defects and randomize the graph. An example is shown in Fig. 4(a).

In the second type, the graph was generated by progressively joining nearby nodes randomly scattered inside a 3D sphere so that each node has a maximum of three links. To begin, we randomly distributed $N$ nodes inside a 3D sphere. We identify the node closest to the center of the sphere, and we link it to the three closest nodes. We then identify the nodes with number of neighbors between 1 and 2 , identify the nodes with less than three neighbors that are the closest to them, and join them. We iterate and terminate the algorithm when no more links were possible (at most, one node has degree 2). Links longer than $70 \%$ of the network geometrical diameter were removed, and the network was given a "haircut," removing all bridges and possible isolated components. Finally, any nodes $k$ connected only to two other nodes $i$ and $j$ were removed, and the links $\langle i k\rangle$ and $\langle j k\rangle$ were replaced by a link $\langle i j\rangle$. Except for the network haircut, the last few steps were meant to simplify the graph without loss of generality. This algorithm is meant to emulate the topology of the network that grows and bifurcates from a central point, much like a growing vascular network. An example of a network produced with this algorithm is shown in Fig. 4(b). The weight of the links in Fig. 4(b) has been assigned randomly. 
The genus 2 torus was intended as a test case of our algorithm in graphs that are easily embeddable and where the generator basis vectors are already known, whereas the random 3D topology was intended as a test case for naturally occurring graphs where typically the embedding is not known. The size of the graphs generated by these procedures fell into two classes: the 500 class (size ranging from $N=478$ to 538 nodes) and the 800 class (size ranging from $N=800$ to 960 nodes).

We used three main generating functions to assign the weights (in particular, the weight order) to the underlying graph topologies. In the first function, termed "random" in the following text, the weights are assigned at random [see, e.g., Fig. 8(a)]. This random, high-disorder assignment was intended as the baseline control case to compare against our more ordered graphs.

For the second function, termed "lines" [Fig. 8(b)], we aimed to emulate a weight distribution with some longrange order, a distribution that would generate linear segments randomly placed on the graph. The initial link $e_{0}$ is chosen at random and assigned the highest weight. Starting from that link, we iteratively transverse a randomly chosen trail on the graph, assigning weights in descending order. When the trail reaches a node with outgoing links that have all been visited and have assigned weights, the trail terminates and a new trail is generated at another randomly selected edge with unassigned weight. At the initial stages of the weight assignment, trails terminate mostly through self-intersections.

The third generating function was similar to the lines model, with the addition of self-avoidance [Fig. 8(c)]. Namely, the tip of the growing trail cannot intersect the last 40 nodes added to the trail. In addition, in this model, termed "self-avoiding (SA) lines," we implemented a length cutoff, and a trail can be at most 12 links long.
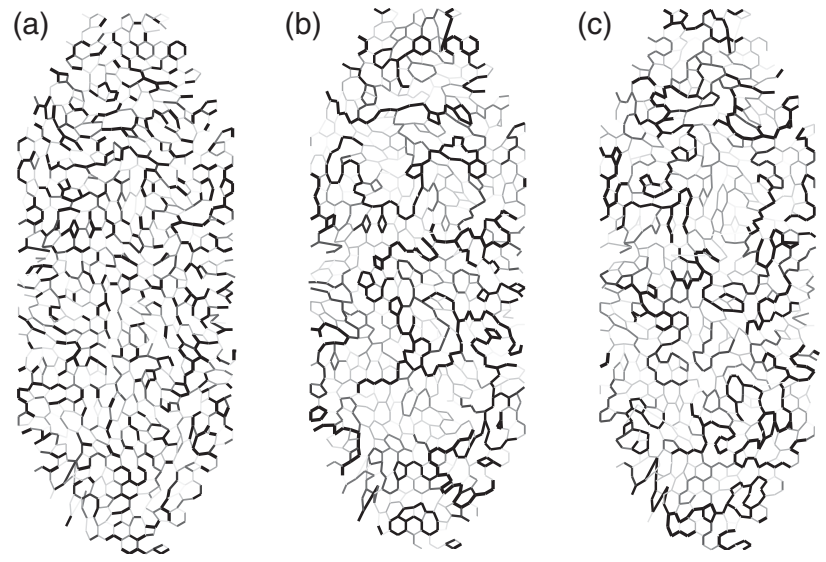

FIG. 8. Examples of different edge-weight assignment functions on a two-holed torus. (a) Random. (b) Lines, no selfavoidance. (c) Lines with SA. Note the high similarity between (b) and (c).
Last, we explored weight assignments produced by a positive feedback adaptation algorithm described in Ref. [33] as a more biologically relevant test case. For more details about the adaptation algorithm, the interested reader can consult Ref. [34]. For completeness, we briefly describe the algorithm here and provide some further detail in the Appendix.

In our adaptive model, each pair of vertices of the network can act as a net current source and sink. The network carries the load from the source to the sink, and the conductivity of the links grows or shrinks according to the average flow through them (the average is being performed over all pairs of vertices that act as sources and sinks). Starting from a random assignment of edge conductivities, the networks evolve and finally converge to a hierarchically organized architecture. This architecture has been shown to be functionally advantageous as it minimizes the weighted path length for all point-to-point transit [33].

By appropriate nondimensionalization, we may reduce the control parameters for our dynamical, adaptive system to the load on the system, $\vartheta$, and $\gamma$, the sigmoidal exponent that controls the strength and sharpness of the feedback. In the simulations shown in this work, the transportation load is $\vartheta=10$, and $\gamma=0.3$ (model termed "adapted 0.3") and $\gamma=0.8$ (model termed "adapted 0.8 "). The underlying topology was random, and the simulation was initialized with various random conductivity value sets $C_{i j}(0)$.

\section{B. Tiles and generators}

With this set of example networks in hand, we can examine the correspondence between the generators of the minimal weight basis and the "true" tiles of some simple spatially embedded networks. In the case of networks with a known topology, this correspondence is clear and exact, with Figs. $9\left(a_{1}\right)$ and $9\left(a_{2}\right)$ showing, for a toroidal network, the raw initial counts of the basis vector length and the counts once the basis set has been rotated onto the minimal weight representation, respectively. As expected, the topological basis elements are much longer than the tiles, and a clear separation develops. A similar effect is observed for a network embedded in a 2-torus [Figs. $9\left(b_{1}\right)$ and $9\left(b_{2}\right)$ ]. In a complex embedded network with an a priori unknown topology, however, the situation is not so clear cut. As Figs. $9\left(c_{1}\right)$ and $9\left(c_{2}\right)$ demonstrate, a randomly embedded $3 \mathrm{D}$ network will have many generators that are clearly tiles, but it may have no strong dividing line between the tiles and topological basis elements. We will show in the next section that even in this worst-case scenario, a great deal of new information can be extracted from these networks with our approach. Finally, in Figs. $9\left(d_{1}\right)$ and $9\left(d_{2}\right)$, we show an example of real data, drawn from a scale-free transport network that we will discuss in Sec. IV D.

In order to better quantify the degree to which these distributions can be used to unambiguously identify the 

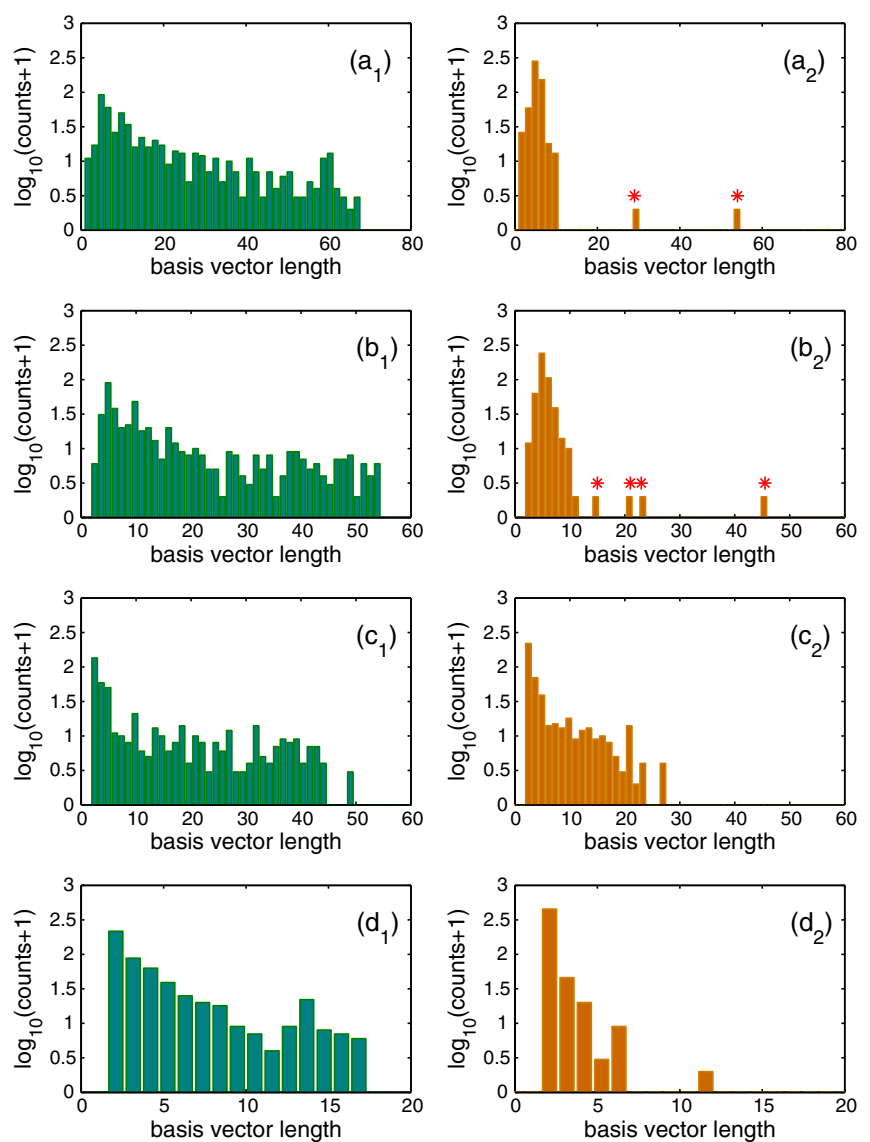

FIG. 9. Raw, untransformed basis vector length counts (left column, green histograms) and the result of rotating these vectors onto the minimal weight basis (right column, orange histograms). Histograms are shown for a network on a torus $\left(a_{i}\right)$, a network on a 2-torus $\left(b_{i}\right)$, a network randomly embedded into space $\left(c_{i}\right)$, and a real transport network $\left(d_{i}\right)$.

topological cycles versus the tiles, we combine Eq. (1) with the relation $|p| f=2 e$ and arrive at an expression for $|p|$ as a function of $v, e$, and $g$ :

$$
|p|=\frac{2 e}{e-v-2 g+2} .
$$

Since $e$ and $v$ for a given network are known (and constant), we can sequentially remove more and more of the longest cycles and compare $p_{m}$, the average number of cycles per basis element of the remaining set of vectors, to the theoretical value given in Eq. (7), with $g$ set equal to half the number of cycles removed. As shown in Fig. 10, when $\left|\left(p_{m}-p_{t h}\right) / p_{t h}\right|$ is equal to zero, then we have correctly identified the tiles. In the cases where zero is never quite achieved, such as the random $3 \mathrm{D}$ embedding, it is likely that the minimum of $\left|\left(p_{m}-p_{t h}\right) / p_{t h}\right|$ is still picking most of the correct topological cycles, but a small number of tiles are larger than the smallest topological cycles and are picked up by the simple procedure of removing longest cycles first. Additionally, it can be the case that $\left|p_{m}\right|$ and

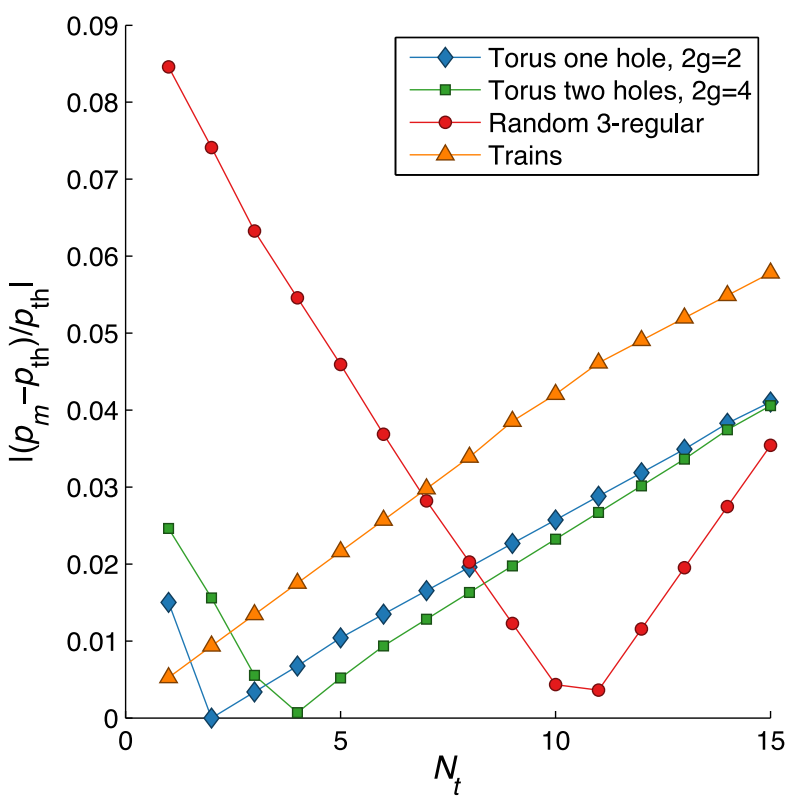

FIG. 10. A comparison of $p_{m}$, the observed average number of links per cycle upon removing the $N_{t}$ largest cycles from the set, and $p_{t h}$, the theoretically predicted value for the given network with genus $g=N_{t} / 2$. When $\left|p_{m}-p_{t h}\right|$ is identically zero, there is an exact match between the removed cycles and the "true" topological cycles. In cases where zero is not achieved, there is some mixing of tiles and topological cycles somewhere in the $N_{t}$-removed cycles. This mixing occurs statistically among the smallest of the removed cycles. Even so, the minimum of this value indicates that many of the cycles are identified correctly.

$\left|p_{t h}\right|$ do not exactly match even when all cycles are identified correctly. This possibility arises from the discrepancy between the total set of faces, which enters $\left|p_{t h}\right|$ as $f$ in the relation $|p| f=2 e$, and the total set of (trial) tile basis vectors used to calculate $\left|p_{m}\right|$, which excludes the single largest face but otherwise matches $f$. Again, we stress that a fully reliable, exact procedure for separating the tiles from the generators of the fundamental group is not feasible; our method is capable of producing exact results some of the time, and in the cases where it does not, it still identifies many of the topological cycles correctly.

\section{Quantifying the results}

We applied the cycle-coalescence algorithm to the example networks and calculated the topological asymmetry $A$ and the average vein length $L$, as described above. In this section, we describe the results of the analysis and demonstrate the power of the algorithm.

In Fig. 11, for random 3D spatial network topologies, we plot the topological asymmetry $A$ versus the average vein length $L$ for five different weight assignment models (random, SA lines, lines, adapted $\gamma=0.5$, and adapted $\gamma=0.8$ ). Each dot in Fig. 11 is a different realization of the weight distribution and underlying topology generation models. In all cases, the underlying graph topology was 


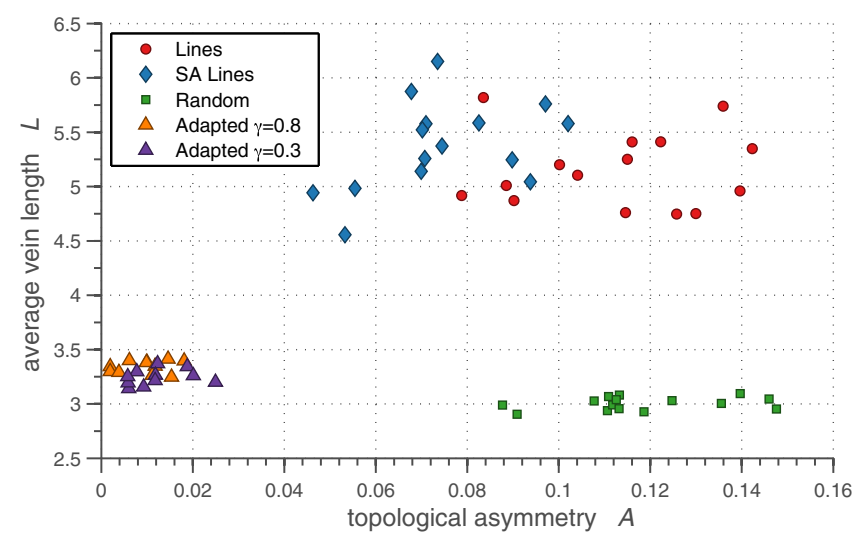

FIG. 11. Average vein length versus topological asymmetry for various edge-weight assignment models on random 3-regular graph topologies. The number of nodes on each graph was approximately $N \simeq 500$. Blue: SA lines; red: lines; green: random; orange: adapted 0.8 ; and purple: adapted 0.5 .

random, and the network sizes were from the 500 class. We see that the topological asymmetry and underlying graph topology can distinguish all the models except the adapted 0.8 and adapted 0.3. Surprisingly, the algorithm can even distinguish the lines from the SA lines, two models that are only subtly different [see Figs. 8(a) and 8(b). Note that $A$ alone would not be able to distinguish the lines model from the random model. The frequent self-intersection of the "veins" in the lines model create a nested cycle architecture that is very similar to the random model at a local level. However, examining $L$, a quantity that captures more longrange information about the graph, we see that the two models are clearly distinguishable. Note also that $L$ alone would not be able to distinguish the lines from the SA lines model. In this case, the two models generate "veins" that have approximately the same average length. However, locally, the SA lines model is more highly nested as it lacks the small cycles that are the results of self-intersections, and the models generate different values for $A$. The random model, having the edge weights assigned at random, lacks any hierarchical organization and nested loops, and has very short vein lengths, placing it at the bottom right of the vein length-asymmetry plot.

Although the topological asymmetry and the average vein length are generically size-dependent quantities, their size dependence is not strong, at least for the models we examined. In Fig. 12, we plot $L$ and $A$ for lines, SA lines, and random models for the two size classes, 500 and 800 . The different size networks generated by the same weight assignment function are indistinguishable, despite the relatively large difference in graph size.

In all the above cases, the underlying network topology was random. We repeat the same procedure for a two-holed torus and see again that the results are qualitatively the same (Fig. 13). Lines and SA lines are cleanly distinguishable but still obviously related, whereas random is

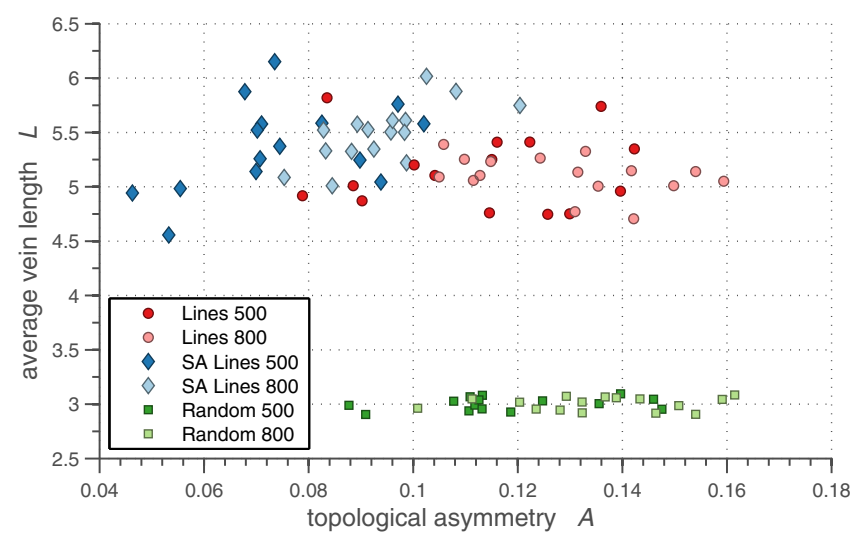

FIG. 12. Average vein length $L$ versus topological asymmetry $A$ for various edge-weight assignment models on random 3 -regular graph topologies. The number of nodes on each graph was approximately $N \simeq 500$ or $N \simeq 800$. Blue: SA lines 500; light blue: SA lines 800; red: lines 500; pink: lines 800; green: random 500; and light green: random 800. $L$ and $A$ are not sensitive to the graph size.

completely separate. Again, the 500 and 800 class networks remain identical.

Table I presents the mean and variance of the point clouds in Figs. 12 and 13. The point clouds are distinguishable, as the variances in $L$ and $A$ are smaller than the separations of the means. However, if a random basis instead of the minimal basis is used, although the $L$ measurements are not affected, the variance in $A$ increases considerably. The point clouds of SA lines and lines start overlapping, and the method stops being able to distinguish the architectures (see Table II).

The cycle-coalescence algorithm can also be used to investigate the evolving architecture of the adaptive model.

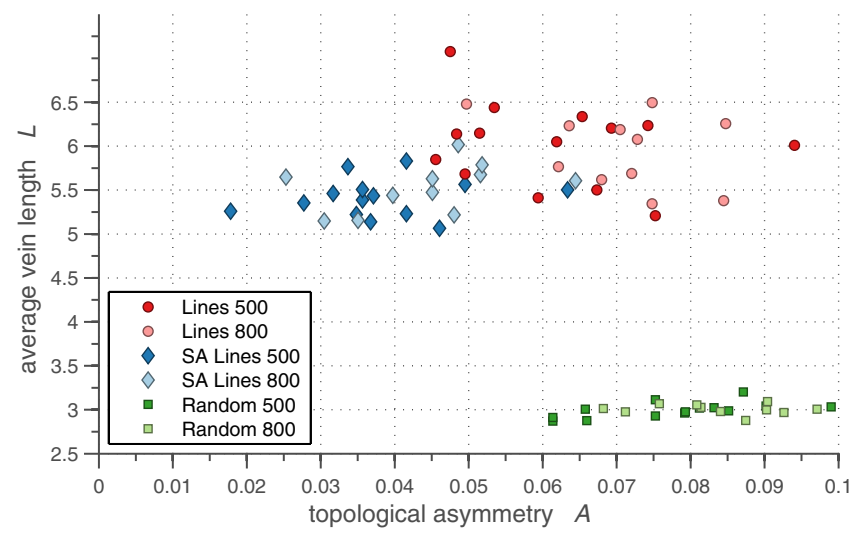

FIG. 13. Average vein length $L$ versus topological asymmetry $A$ for various edge-weight assignment models on genus-2 toroidal topologies. The number of nodes on each graph was approximately $N \simeq 500$ or $N \simeq 800$. Blue: SA lines 500; light blue: SA Lines 800; red: lines 500; pink: lines 800; green: random 500; light green: and random 800. $L$ and $A$ are not sensitive to the graph size. 
TABLE I. Mean and variance of $A$ and $L$ of the point clouds in Figs. 12 and 13.

\begin{tabular}{lcc}
\hline \hline Lines 500 & $0.113 \pm 0.021$ & $5.15 \pm 0.34$ \\
SA lines 500 & $0.075 \pm 0.016$ & $5.37 \pm 0.41$ \\
Random 500 & $0.118 \pm 0.018$ & $3.004 \pm 0.058$ \\
Lines 800 & $0.129 \pm 0.018$ & $5.11 \pm 0.18$ \\
SA lines 800 & $0.094 \pm 0.011$ & $5.49 \pm 0.27$ \\
Random 800 & $0.136 \pm 0.017$ & $3.003 \pm 0.061$ \\
2-Torus, min basis & $A \pm d A$ & $L \pm d L$ \\
Lines 500 & $0.062 \pm 0.014$ & $6.02 \pm 0.48$ \\
SA lines 500 & $0.038 \pm 0.011$ & $5.41 \pm 0.22$ \\
Random 500 & $0.078 \pm 0.011$ & $2.997 \pm 0.090$ \\
Lines 800 & $0.071 \pm 0.010$ & $5.96 \pm 0.42$ \\
SA lines 800 & $0.044 \pm 0.011$ & $5.53 \pm 0.27$ \\
Random 800 & $0.084 \pm 0.009$ & $3.006 \pm 0.058$ \\
\hline \hline
\end{tabular}

Unsurprisingly, we discover a clear progression through our characteristic space from the unadapted, randomly weighted initial networks to final states with drastically lower topological asymmetry and slightly higher vein length [Fig. 14(a)]. Interestingly, however, despite the fact that varying the exponent in the sigmoidal of the adaptation's feedback function does not seem to affect where in our characteristic space the networks end up, by looking at intermediate stages of the adaptation, one can clearly see that the higher-exponent sample is much closer to its finalstate architecture; thus, these networks are adapting "faster" in a real sense. Despite the power evident in the choice of characterizing statistics that we have chosen for the characteristic tree, we reiterate that not all information is being captured by these two probes. For example, the weight distribution in the final adapted state is very different for the two different values of $\gamma$ [Fig. 14(b)]. Our method is only sensitive to the sort order of the weights

TABLE II. Mean and variance of $A$ for the same networks as in Table I, now calculated using a random rather than the minimal basis. The $L$ values are not affected by a shift in basis, so they are not included in the table.

Lines 500

SA lines 500

Random 500

Lines 800

SA lines 800

Random 800

\section{2-Torus, random basis}

Lines 500

SA lines 500

Random 500

Lines 800

SA lines 800

Random 800
$0.078 \pm 0.014$

$0.066 \pm 0.013$

$0.085 \pm 0.016$

$0.100 \pm 0.012$

$0.090 \pm 0.011$

$0.111 \pm 0.014$

$$
A \pm d A
$$

$0.057 \pm 0.010$

$0.055 \pm 0.013$

$0.064 \pm 0.011$

$0.087 \pm 0.014$

$0.075 \pm 0.014$

$0.082 \pm 0.011$
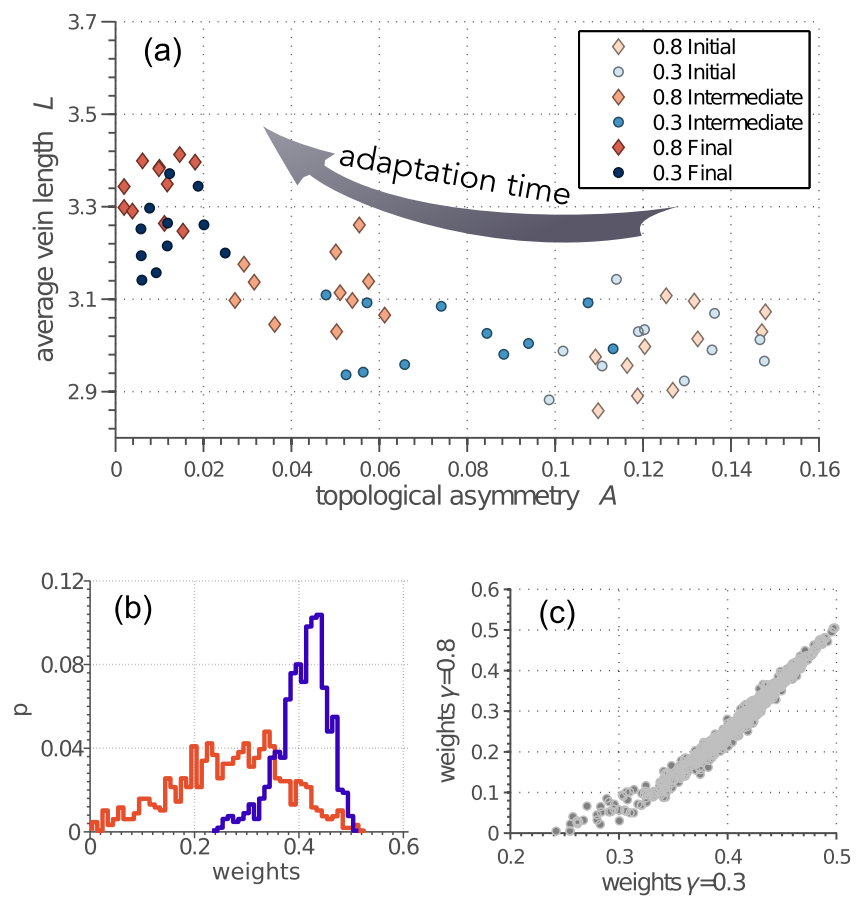

FIG. 14. (a) Average vein length $L$ versus topological asymmetry $A$ for initial, intermediate, and final states of an initially random-weighted network evolving under our adaptive model. (b) Distribution of the edge weights in the final adapted states for two different sigmoidal-feedback control exponents, $\gamma$. (c) Structural correlations in the adapted weights from identical starting configurations for two different sigmoidal-feedback control exponents, $\gamma$.

and not the full weight distribution. The two fully adapted graphs $\gamma=0.3$ and $\gamma=0.8$ thus have very different weight distributions but very similar architecture, which could not have been guessed from information about the weights alone. Interestingly, starting from the same underlying topology and running the adaptation for the two different gammas, we see that $C_{i, j}^{\gamma=0.8}$ correlates strongly with $C_{i, j}^{\gamma=0.3}$ [Fig. 14(c)], pointing to some kind of universal hierarchical organization for this system. Interestingly, this hierarchically nested architecture has been shown to optimize the transport properties of the system. It remains to be shown whether all transport networks with low topological asymmetry share this feature.

\section{Real-world networks}

Last, we tested how the cycle-coalescence algorithm performs in real-world weighted network data that do not have fixed or approximately fixed degree. We used data from the Multilayer Temporal Network of Public Transport in Great Britain, produced by Riccardo Gallotti of the QuantURB group [35]. This data set describes the transport Network of Great Britain using a multilayer node list and edge list, where each layer describes a mode of transport. For our analysis, we used the train and coach layers. The 
nodes of these two layers correspond to train and coach stations, and the data set provides information about the distance between each pair of connected nodes and the travel time between them. We assigned a weight to each edge that was proportional to the speed with which this particular link is traversed (distance over time), and trimmed any tree segments of the networks. To bring the networks to sizes comparable to the data presented in the rest of the work (approximately $10^{3}$ nodes), we thresholded the edges based on their weight, disregarding approximately $5 \%$ of the edges of the coach network and $30 \%$ of the train network. Finally, without loss of generality, we "bypassed" any stations that were not hubs, namely, any stations that were only connected to two other stations. As a result, the remaining stations in the analyzed graphs still contained all the information about the nested cycles, but all the stations had degree of at least 3 .

To test the sensitivity of our results to thresholding, we generated an additional network from the train data set with a higher threshold. Our coach network contained 299 nodes, the train network 414, and the train high-threshold network 331. In Fig. 15(a), we plot the average topological length $L$ versus the topological asymmetry $A$ for the three networks - the coach network (large green square), and the low- and high-threshold train networks (large blue diamond and large red circle, respectively). Our results are robust to thresholding, as the low- and high-threshold train networks are very close compared to the coach network. The train and coach networks appear in very different areas of the vein length vs asymmetry plot. However, the underlying, unweighted topology of the coach and train networks is different, and to understand the information in Fig. 15(a), we need to decouple the effect of the edge weights on $L$ and $A$ from the topology itself. To do this, we generated 50 additional data sets for each of the three underlying topologies (coach, train, and train high threshold) by randomizing the edge weights. We plot these data on Fig. 15(a) (small green, blue, and red symbols, respectively). We see that the United Kingdom coach system has topologically longer routes than a network with random assignment of the weights, but lower nestedness [see also Fig. 7(a), top right]. On the contrary, the train network has relatively short routes but is more nested than what a random model would predict [see also Fig. 7(a), bottom left]. We believe that these data reflect the fact that coach routes are not constrained by preexisting railway tracks and, for this reason, favor a less gridlike topology, but more work is needed to substantiate this interpretation.

Our methodology provides insights for a system far from the constrained degree networks where we have validated our metrics. As can be seen in Fig. 15(b), the degree distribution for the coach and train networks resembles a power law, unlike the fixed degree networks we examined in the previous sections. It is possible that for certain topologies that do not have a fixed degree, the minimum
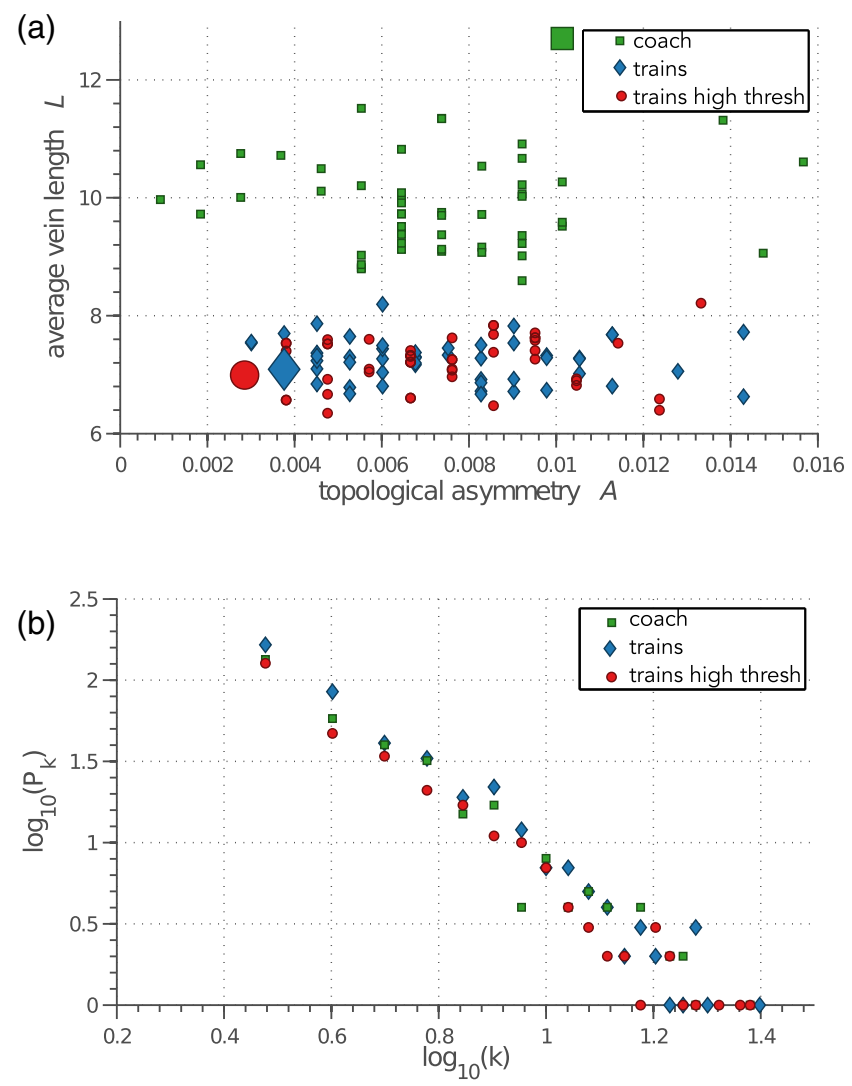

FIG. 15. (a) Average vein length $L$ versus topological asymmetry $A$ for the coach network (large green square), and the lowand high-threshold train one (large blue diamond and red circle, respectively). The small symbols represent networks with the same underlying topology as the coach network (small green squares), and the low- and high-threshold train one (small blue diamond and small red circle, respectively), but randomized edge weights. (b) Degree distribution of the topologies of the coach and train networks. The numbers of nodes $P_{k}$ of degree $k$ can be approximated by a power law.

weight cycle basis is still a good starting point for the cycle-coalescence algorithm. Future work will examine these aspects.

\section{DISCUSSION}

We have presented a well-defined, statistically robust algorithm to characterize the structure and topology of weighted networks in three space dimensions. This method exactly matches known techniques in two dimensions and thus represents a natural extension and generalization of these earlier methods. Our quantification method can be used to understand the underlying architecture of 3D graphs in a way not possible before, as we have shown in the example of the adapting networks, where the $\gamma=0.3$ and $\gamma=0.8$ graphs may have wildly different weight distributions but are revealed to be structurally similar nonetheless. Indeed, working with traditional classification 
methods that attempt to leverage primarily edge-weight families has recently shown to be necessarily incomplete [24,36]. Additionally, the inclusion of some kind of topological information to supplement that weight information is known to be desirable. Our cycle-coalescence algorithm represents just such a tool but includes sensitivity to the geometry of the weight placement as well—given networks with the same underlying topology and weights drawn from the same distribution, it is easily possible to construct examples that functionally have very different architecture that no previously extant characteristic could distinguish. We reiterate that while the relevance of the abstract mapping of the network to a low-genus surface is intuitive and clearly powerful, a deep, underlying understanding of exactly what physical or biological attributes of these systems are laid bare by this mapping is still an important question for future work.

Furthermore, our characteristic prescription is insulated against noise, as we have demonstrated only weak to nonexistent sensitivity to simple variance in graph size, while the doubly statistical nature of the tool protects against tile misidentification. We point out that such misidentification must always remain a possibility for any algorithm that is expected to run in reasonable time for even moderately sized networks, as the complete removal of the possibility of tile misidentification would represent a solution to the NP-hard graph-genus problem.

The presentation of the algorithm adopts a language that assumes that the shortest basis cycles constitute a tiling of an embedding of the surface. Choosing the shortest cycle basis as our minimal basis is important, as short cycles contain edges that are topologically close by. A shortest cycle basis as a starting point of our cycle-coalescence algorithm ensures that the higher branches of the characteristic tree (branches that are furthest from the tree root) describe the architecture of local neighborhoods of the graph. Although there is always the possibility of a degeneracy in the minimal basis, i.e., two different bases with the same total number of edges, we do not expect that the exact choice of minimal basis will affect the results significantly, as all the minimal bases should be good descriptors for the local neighborhoods. However, a different prescription for choosing the tiles might be appropriate for different classes of networks that have unusual edgeweight distributions or are not 3-regular. Similarly, an extension of the algorithm is needed to treat networks that have treelike components or, in general, bridges (edges whose deletion creates disconnected components).

There has even been recent interest in the study of graphs and networks that live embedded in a given complex surface from the start [37]. Our work represents a pathway to bring the developments and techniques designed for the study of these networks to bear on all 3D networks.

It is our belief that this cycle-coalescence characterization of three-dimensional networks will find wide applicability across many physical and biological representatives, hopefully uncovering new ways of thinking about these systems. From organ vasculature to neural networks, ant farms or hyphal networks to root networks of clonal colonies like quaking aspen, force networks in sand piles to airline routing, new descriptive and predictive modeling is possible.

\section{ACKNOWLEDGMENTS}

This work was supported in part by NSF under Grant No. PHY-1058899. E. K. wishes to thank the Burroughs Wellcome Fund.

\section{APPENDIX: NETWORK EVOLUTION MODEL}

The network evolution model we have chosen to use here is governed by a system of equations, describing transport, conservation, and adaptation. The model is explained in detail in Ref. [33]. The flow in the network is considered to be Hagen-Poiseuilleian laminar tube flow, so the transport equation for each edge is simply

$$
Q_{i j}^{k l}=C_{i j} \cdot\left(p_{i}^{k l}-p_{j}^{k l}\right) .
$$

$Q_{i j}^{k l}$ is the flow through the edge $\{i, j\}$, when node $k$ is a source and node $l$ a sink. The hydrostatic pressure difference $\Delta p_{i j}^{k l}:=p_{i}^{k l}-p_{j}^{k l}$ along the tube between the pressures $p_{i}^{k l}$ and $p_{j}^{k l}$ defined at the nodes $i$ and $j$ acts as a potential difference from which the flow arises. The proportionality factor is the conductivity of the tube, denoted as $C_{i j}$.

Meanwhile, from flow conservation at each node $i$ and the current boundary conditions, we have

$$
\sum_{j, \forall\{i, j\} \in \mathbb{E}} Q_{i j}^{k l}=\left(\delta_{i k}-\delta_{i l}\right) \cdot \zeta,
$$

where $\mathbb{E}$ is the set of all edges. For each node, the sum of incoming flows must equal the sum of outgoing flows, unless the node is a source or sink that contributes an additional flow, $\zeta \geq 0$.

The ensemble averaged mean flow is

$$
\left\langle\left|Q_{i j}\right|\right\rangle:=\frac{1}{\frac{N \cdot(N-1)}{2}} \sum_{(k, l) \in \mathbb{P}}\left|Q_{i j}^{k l}\right|,
$$

where $\mathbb{P}$ is the set of all node pairs, and flows are considered equally in both directions.

Finally, we model the adaptation process with a differential equation describing the time evolution of the conductivities $C_{i j}=C_{i j}(t)$ :

$$
\frac{\mathrm{d} C_{i j}(t)}{\mathrm{d} t}=\beta \cdot f\left(\frac{\left\langle\left|Q_{i j}(t)\right|\right\rangle}{\epsilon}\right)-\alpha \cdot C_{i j}(t) .
$$


This equation features a positive, nonlinear feedback term $\beta \cdot f\left(\left\langle\left|Q_{i j}(t)\right|\right\rangle / \epsilon\right)$ that grows an edge's conductivity as a function of the scaled mean flow $\left\langle\left|Q_{i j}(t)\right|\right\rangle / \epsilon$ through itself. Balancing this term is a negative, exponential decay term $-\alpha \cdot C_{i j}(t)$. The parameters $\beta \geq 0$ and $\epsilon>0$ scale the feedback and the flow through one edge; $\alpha \geq 0$ is the exponential decay parameter. The feedback function is sigmoidal, $f(x)=\left[x^{\gamma} /\left(1+x^{\gamma}\right)\right]$.

At each step of the algorithm, we calculate $\left\langle\left|Q_{i j}\right|\right\rangle$ for a particular set of conductivities and then apply Eq. (A4) to get a new, updated set of conductivities. We evolve this model until it reaches a steady state, and the conductivities attain their final equilibrium values.

[1] D. J. Watts and S.H. Strogatz, Collective Dynamics of "Small-World" Networks, Nature (London) 393, 440 (1998).

[2] A. Barabási and R. Albert, Emergence of Scaling in Random Networks, Science 286, 509 (1999).

[3] H. Seybold, J. S. Andrade, and H. J. Herrmann, Modeling River Delta Formation, Proc. Natl. Acad. Sci. U.S.A. 104, 16804 (2007).

[4] I. Rodriguez-Iturbe and A. Rinaldo, Fractal River Basins: Chance and Self-Organization (Cambridge University Press, Cambridge, England, 1997).

[5] E. Katifori and M.O. Magnasco, Quantifying Loopy Network Architectures, PLoS One 7, e37994 (2012).

[6] Y. Mileyko, H. Edelsbrunner, C. A. Price, and J. S. Weitz, Hierarchical Ordering of Reticular Networks, PLoS One 7, e36715 (2012).

[7] H. Ronellenfitsch, J. Lasser, D. C. Daly, and E. Katifori, Topological Phenotypes Constitute a New Dimension in the Phenotypic Space of Leaf Venation Networks, PLoS Comput. Biol. 11, e1004680 (2015).

[8] A. Perna, P. Kuntz, and S. Douady, Characterisation of Spatial Network-like Patterns from Junctions' Geometry, Phys. Rev. E 83, 066106 (2011).

[9] L. M. Escudero, L. da F Costa, A. Kicheva, J. Briscoe, M. Freeman, and M. M. Babu, Epithelial Organisation Revealed by a Network of Cellular Contacts, Nat. Commun. 2, 526 (2011).

[10] C. B. Schaffer, B. Friedman, N. Nishimura, L. F. Schroeder, P. S. Tsai, F. F. Ebner, P. D. Lyden, and D. Kleinfeld, Two-Photon Imaging of Cortical Surface Microvessels Reveals a Robust Redistribution in Blood Flow after Vascular Occlusion, PLoS Biol. 4, e22 (2006).

[11] P. Blinder, A. Y. Shih, C. Rafie, and D. Kleinfeld, Topological Basis for the Robust Distribution of Blood to Rodent Neocortex, Proc. Natl. Acad. Sci. U.S.A. 107, 12670 (2010).

[12] M. Fernández, D. Semela, J. Bruix, I. Colle, M. Pinzani, and J. Bosch, Angiogenesis in Liver Disease, J. Hepatol. 50, 604 (2009).

[13] O. Veblen, An Application of Modular Equations in Analysis Situs, Ann. Math. 14, 86 (1912).

[14] J. L. Gross and J. Yellen, Graph Theory and Its Applications (CRC Press, Boca Raton, FL, 2005).
[15] W. Kocay and D. L. Kreher, Graphs, Algorithms, and Optimization, Vol. 29 (CRC Press, Boca Raton, FL, 2004).

[16] O. Giménez and M. Noy, Asymptotic Enumeration and Limit Laws of Planar Graphs, J. Am. Math. Soc. 22, 309 (2009).

[17] F. Bernhart and P. C. Kainen, The Book Thickness of a Graph, J. Comb. Theory B 27, 320 (1979).

[18] C. Thomassen, The Graph Genus Problem Is NP-Complete, J. Algorithms 10, 568 (1989).

[19] H. Poincaré, Analysis situs, J. l'École Polytech. 1, 1 (1895).

[20] A. Hatcher, Algebraic Topology (Cambridge University Press, Cambridge, England, 2002).

[21] J. D. Horton, A Polynomial-Time Algorithm to Find the Shortest Cycle Basis of a Graph, SIAM J. Comput. 16, 358 (1987).

[22] E. Katifori, G. J. Szöllösi, and M. O. Magnasco, Damage and Fluctuations Induce Loops in Optimal Transport Networks, Phys. Rev. Lett. 104, 048704 (2010).

[23] M. E. J. Newman, The Structure and Function of Complex Networks, SIAM Rev. 45, 167 (2003).

[24] A. Barrat, M. Barthelemy, R. Pastor-Satorras, and A. Vespignani, The Architecture of Complex Weighted Networks, Proc. Natl. Acad. Sci. U.S.A. 101, 3747 (2004).

[25] W. Risau, Mechanisms of Angiogenesis, Nature (London) 386, 671 (1997).

[26] H. S. M. Coxeter, The Problem of Packing a Number of Equal Nonoverlapping Circles on a Sphere, Trans. N.Y. Acad. Sci. 24, 320 (1962).

[27] D. L. Weaire and S. Hutzler, The Physics of Foams (Oxford University Press, New York, 2000).

[28] F. Sausset and G. Tarjus, Periodic Boundary Conditions on the Pseudosphere, J. Phys. A 40, 12873 (2007).

[29] R. E. Horton, Erosional Development of Streams and Their Drainage Basins-Hydrophysical Approach to Quantitative Morphology, Geol. Soc. Am. Bull. 56, 275 (1945).

[30] A. N. Strahler, Hypsometric (Area-Altitude) Analysis of Erosional Topography, Geol. Soc. Am. Bull. 63, 1117 (1952).

[31] J. VanPelt, H. B. M. Uylings, R. W. H. Verwer, R. J. Pentney, and M. J. Woldenberg, Tree Asymmetry-A Sensitive and Practical Measure for Binary Topological Trees, Bull. Math. Biol. 54, 759 (1992).

[32] https://github.com/hronellenfitsch/nesting.

[33] J. Gräwer, C. D. Modes, M. O. Magnasco, and E. Katifori, Structural Self-Assembly and Glassy Dynamics in Locally Adaptive Networks, Phys. Rev. E 92, 012801 (2015).

[34] A. Tero, T. Saigusa, R. Kobayashi, and T. Nakagaki, Rules for Biologically Inspired Adaptive Network Design, Science 327, 439 (2010).

[35] R. Gallotti and M. Barthelemy, The Multilayer Temporal Network of Public Transport in Great Britain, Scientific Data 2, 140056 (2015).

[36] L. F. Costa, F. A. Rodrigues, G. Travieso, and P. R. Villas Boas, Characterization of Complex Networks: A Survey of Measurements, Adv. Phys. 56, 167 (2007).

[37] T. Aste, R. Gramatica, and T. Di Matteo, Exploring Complex Networks via Topological Embedding on Surfaces, Phys. Rev. E 86, 036109 (2012). 